\title{
Betweenness Centrality in Some Classes of Graphs
}

\author{
Sunil Kumar Raghavan Unnithan, Balakrishnan Kannan, and Madambi Jathavedan
}

Department of Computer Applications, Cochin University of Science and Technology, Cochin 682022, India

Correspondence should be addressed to Sunil Kumar Raghavan Unnithan; sunilstands@gmail.com

Received 21 May 2014; Accepted 21 October 2014; Published 25 December 2014

Academic Editor: Chris A. Rodger

Copyright (c) 2014 Sunil Kumar Raghavan Unnithan et al. This is an open access article distributed under the Creative Commons Attribution License, which permits unrestricted use, distribution, and reproduction in any medium, provided the original work is properly cited.

\begin{abstract}
There are several centrality measures that have been introduced and studied for real-world networks. They account for the different vertex characteristics that permit them to be ranked in order of importance in the network. Betweenness centrality is a measure of the influence of a vertex over the flow of information between every pair of vertices under the assumption that information primarily flows over the shortest paths between them. In this paper we present betweenness centrality of some important classes of graphs.
\end{abstract}

\section{Introduction}

Betweenness centrality plays an important role in analysis of social networks [1,2], computer networks [3], and many other types of network data models [4-9].

In the case of communication networks the distance from other units is not the only important property of a unit. What is more important is which units lie on the shortest paths (geodesics) among pairs of other units. Such units have control over the flow of information in the network. Betweenness centrality is useful as a measure of the potential of a vertex for control of communication. Betweenness centrality [10-14] indicates the betweenness of a vertex in a network and it measures the extent to which a vertex lies on the shortest paths between pairs of other vertices. In many real-world situations it has quite a significant role.

Determining betweenness is simple and straightforward when only one geodesic connects each pair of vertices, where the intermediate vertices can completely control communication between pairs of others. But when there are several geodesics connecting a pair of vertices, the situation becomes more complicated and the control of the intermediate vertices gets fractionated.

\section{Background}

The concept of betweenness centrality was first introduced by Bavelas in 1948 [15]. The importance of the concept of vertex centrality is in the potential of a vertex for control of information flow in the network. Positions are viewed as structurally central to the degree to which they stand between others and can therefore facilitate, impede, or bias the transmission of messages. Freeman in his papers $[5,16]$ classified betweenness centrality into three measures. The three measures include two indexes of vertex centrality-one based on counts and one on proportions-and one index of overall network or graph centralization.

2.1. Betweenness Centrality of a Vertex. Betweenness centrality $C_{B}(v)$ for a vertex $v$ is defined as

$$
C_{B}(v)=\sum_{s \neq v \neq t} \frac{\sigma_{s t}(v)}{\sigma_{s t}},
$$

where $\sigma_{s t}$ is the number of shortest paths with vertices $s$ and $t$ as their end vertices, while $\sigma_{s t}(v)$ is the number of those shortest paths that include vertex $v$ [16]. High centrality scores indicate that a vertex lies on a considerable fraction of shortest paths connecting pairs of vertices.

(i) Every pair of vertices in a connected graph provides a value lying in $[0,1]$ to the betweenness centrality of all other vertices.

(ii) If there is only one geodesic joining a particular pair of vertices, then that pair provides a betweenness centrality 1 to each of its intermediate vertices and 
zero to all other vertices. For example, in a path graph, a pair of vertices provides a betweenness centrality 1 to each of its interior vertices and zero to the exterior vertices. A pair of adjacent vertices always provides zero to all others.

(iii) If there are $k$ geodesics of length 2 joining a pair of vertices, then that pair of vertices provides a betweenness centrality $1 / k$ to each of the intermediate vertices.

Freeman [16] proved that the maximum value taken by $C_{B}(v)$ is achieved only by the central vertex in a star as the central vertex lies on the geodesic (which is unique) joining every pair of other vertices. In a star $S_{n}$ with $n$ vertices, the betweenness centrality of the central vertex is therefore the number of such geodesics which is $\left(\begin{array}{c}n-1 \\ 2\end{array}\right)$. The betweenness centrality of each pendant vertex is zero since no pendant vertex lies in between any geodesic. Again it can be seen that the betweenness centrality of any vertex in a complete graph $K_{n}$ is zero since no vertex lies in between any geodesic as every geodesic is of length 1 .

2.2. Relative Betweenness Centrality. The betweenness centrality increases with the number of vertices in the network, so a normalized version is often considered with the centrality values scaled to between 0 and 1 . Betweenness centrality can be normalized by dividing $C_{B}(v)$ by its maximum value. Among all graphs of $n$ vertices the central vertex of a star graph $S_{n}$ has the maximum value which is $\left(\begin{array}{c}n-1 \\ 2\end{array}\right)$. The relative betweenness centrality is therefore defined as

$$
C_{B}^{\prime}(v)=\frac{C_{B}(v)}{\operatorname{Max} C_{B}(v)}=\frac{2 C_{B}(v)}{(n-1)(n-2)} \quad 0 \leq C_{B}^{\prime}(v) \leq 1 .
$$

2.3. Betweenness Centrality of a Graph. The betweenness centrality of a graph measures the tendency of a single vertex to be more central than all other vertices in the graph. It is based on differences between the centrality of the most central vertex and that of all others. Freeman [16] defined the betweenness centrality of a graph as the average difference between the measures of centrality of the most central vertex and that of all other vertices.

The betweenness centrality of a graph $G$ is defined as

$$
C_{B}(G)=\frac{\sum_{i=1}^{n}\left[C_{B}\left(v^{*}\right)-C_{B}\left(v_{i}\right)\right]}{\operatorname{Max} \sum_{i=1}^{n}\left[C_{B}\left(v^{*}\right)-C_{B}\left(v_{i}\right)\right]},
$$

where $C_{B}\left(v^{*}\right)$ is the largest value of $C_{B}\left(v_{i}\right)$ for any vertex $v_{i}$ in the given graph $G$ and $\operatorname{Max} \sum_{i=1}^{n}\left[C_{B}\left(v^{*}\right)-C_{B}\left(v_{i}\right)\right]$ is the maximum possible sum of differences in centrality for any graph of $n$ vertices which occur in star with the value $n-1$ times $C_{B}(v)$ of the central vertex, that is, $(n-1)\left(\begin{array}{c}n-1 \\ 2\end{array}\right)$.

Therefore the betweenness centrality of $G$ is defined as

$$
\begin{aligned}
& C_{B}(G)=\frac{2 \sum_{i=1}^{n}\left[C_{B}\left(v^{*}\right)-C_{B}\left(v_{i}\right)\right]}{(n-1)^{2}(n-2)} \\
& \text { or } C_{B}(G)=\frac{\sum_{i=1}^{n}\left[C_{B}^{\prime}\left(v^{*}\right)-C_{B}^{\prime}\left(v_{i}\right)\right]}{(n-1)} .
\end{aligned}
$$

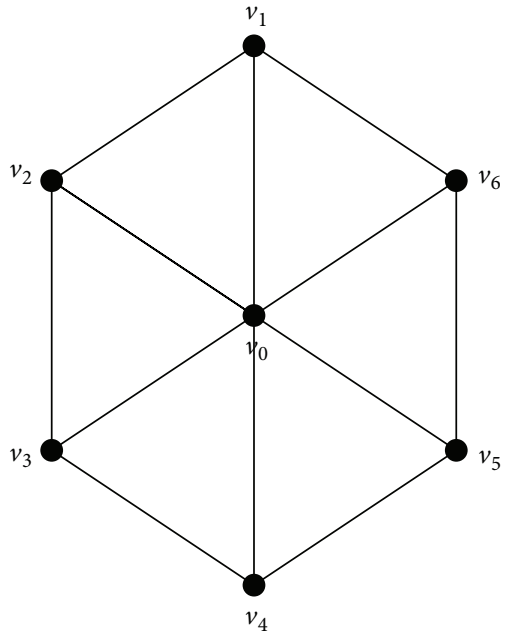

Figure 1: Wheel graph $W_{7}$.

The index, $C_{B}(G)$, determines the degree to which $C_{B}\left(v^{*}\right)$ exceeds the centrality of all other vertices in $G$. Since $C_{B}(G)$ is the ratio of an observed sum of differences to its maximum value, it will vary between 0 and $1 . C_{B}(G)=0$ if and only if all $C_{B}\left(v_{i}\right)$ are equal, and $C_{B}(G)=1$ if and only if one vertex $v^{*}$ completely dominates the network with respect to centrality. Freeman showed that all of these measures agree in assigning the highest centrality index to the star graph and the lowest to the complete graph (see Table 1).

In this paper we present the betweenness centrality measures in some important classes of graphs which are the basic components of larger complex networks.

\section{Betweenness Centrality of Some Classes of Graphs}

\subsection{Betweenness Centrality of Vertices in Wheels}

Wheel Graph $W_{n}$. A Wheel graph $W_{n}$ is obtained by joining a new vertex to every vertex in a cycle $C_{n-1}$. It was invented by the eminent graph theorist W. T. Tutte. A wheel graph on 7 vertices is shown in Figure 1.

Theorem 1. The betweenness centrality of a vertex $v$ in a wheel graph $W_{n}, n>5$, is given by

$$
C_{B}(v)= \begin{cases}\frac{(n-1)(n-5)}{2}, & \text { if } v \text { is the central vertex } \\ \frac{1}{2}, & \text { otherwise. }\end{cases}
$$

Proof. In the wheel graph $W_{n}$ the central vertex is adjacent to each vertex of the cycle $C_{n-1}$. Consider the central vertex of $W_{n}$ for $n>5$. On $C_{n-1}$ each pair of adjacent vertices contributes centrality 0 , each pair of alternate vertices contributes centrality $1 / 2$, and all other pairs contribute centrality 1 to the central vertex. Since there are $n-1$ vertices on $C_{n-1}$, there exist $n-1$ adjacent pairs, $n-1$ alternate pairs, and 
TABLE 1: Graphs showing extreme betweenness.

\begin{tabular}{lccc}
\hline$G$ & $C_{B}(v)$ & $C_{B}{ }^{\prime}(v)$ & $C_{B}(G)$ \\
\hline$S_{n}$ & $\left\{\begin{array}{ccc}(n-1 \\
2\end{array}\right)$ & for central vertex \\
0 & for other vertices & 0 & $\left\{\begin{array}{lll}1 & \text { for central vertex } \\
0 & \text { for other vertices }\end{array}\right.$ \\
\hline$K_{n}$ & 0 & 0 \\
\hline
\end{tabular}

$\left(\begin{array}{c}n-1 \\ 2\end{array}\right)-2(n-1)=(n-1)(n-6) / 2$ other pairs. Therefore the betweenness centrality of the central vertex is given by $(1 / 2)(n-1)+1((n-1)(n-6) / 2)=(n-1)(n-5) / 2$. Now for any vertex on $C_{n-1}$, there are two geodesics joining its adjacent vertices on $C_{n-1}$, one of which passing through it. Therefore the betweenness centrality of any vertex on $C_{n-1}$ is $1 / 2$.

Note. It can be seen easily that $C_{B}(v)=0$ for every vertex $v$ in $W_{4}$ and

$$
C_{B}(v)= \begin{cases}\frac{2}{3}, & \text { if } v \text { is the central vertex } \\ \frac{1}{3}, & \text { otherwise }\end{cases}
$$

in $W_{5}$.

The relative centrality and graph centrality are as follows:

$$
\begin{aligned}
C_{B}^{\prime}(v) & =\frac{2 C_{B}(v)}{(n-1)(n-2)} \\
& = \begin{cases}\frac{(n-5)}{n-2}, & \text { if } v \text { is the central vertex, } \\
\frac{1}{(n-1)(n-2)}, & \text { otherwise, }\end{cases} \\
C_{B}\left(W_{n}\right) & =\frac{\sum_{i=1}^{n}\left[C_{B}^{\prime}\left(v^{*}\right)-C_{B}^{\prime}\left(v_{i}\right)\right]}{(n-1)}=\frac{n^{2}-6 n+4}{(n-1)(n-2)} .
\end{aligned}
$$

3.2. Betweenness Centrality of Vertices in the Graph $K_{n}-e$. A complete graph on 6 vertices with one edge deleted is shown in Figure 2.

Theorem 2. Let $K_{n}$ be a complete graph on $n$ vertices and $e=$ $\left(v_{i}, v_{j}\right)$ an edge of it. Then the betweenness centrality of vertices in $K_{n}-e$ is given by

$$
C_{B}(v)= \begin{cases}\frac{1}{n-2}, & \text { if } v \neq v_{i}, v_{j}, \\ 0, & \text { otherwise. }\end{cases}
$$

Proof. Suppose the edge $\left(v_{i}, v_{j}\right)$ is removed from $K_{n}$. Now $v_{i}$ and $v_{j}$ can be joined by means of any of the remaining $n-2$ vertices. Thus there are $n-2$ geodesics joining $v_{i}$ and $v_{j}$ each containing exactly one vertex as intermediary. This provides a betweenness centrality $1 /(n-2)$ to each of the $n-2$ vertices. Again $v_{i}$ and $v_{j}$ do not lie in between any geodesics and therefore their betweenness centralities are zero.

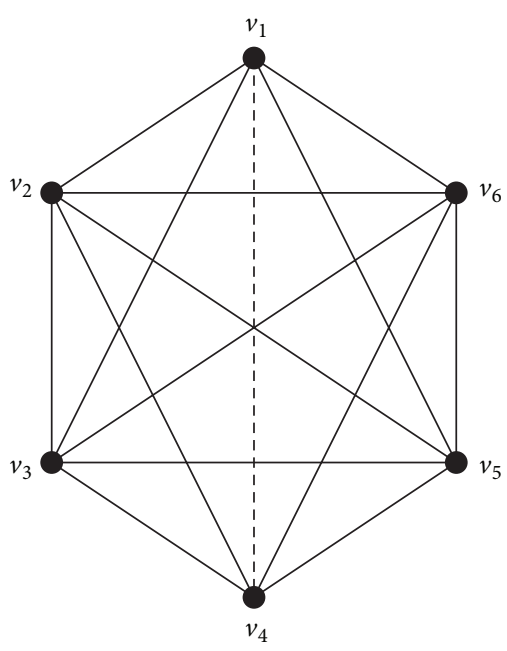

Figure 2: Complete graph $K_{6}-\left(v_{1}, v_{4}\right)$.

The relative centrality and graph centrality are as follows:

$$
\begin{aligned}
C_{B}^{\prime}(v) & =\frac{2 C_{B}(v)}{(n-1)(n-2)} \\
& = \begin{cases}\frac{2}{(n-1)(n-2)^{2}}, & v \neq v_{i}, v_{j} \\
0, & \text { otherwise }\end{cases}
\end{aligned}
$$

$$
C_{B}(G)=\frac{\sum_{i=1}^{n}\left[C_{B}^{\prime}\left(v^{*}\right)-C_{B}^{\prime}\left(v_{i}\right)\right]}{(n-1)}=\frac{4}{(n-1)^{2}(n-2)^{2}} .
$$

\subsection{Betweenness Centrality of Vertices in Complete Bipartite Graphs}

Complete Bipartite Graphs $K_{m, n}$. A graph is complete bipartite if its vertices can be partitioned into two disjoint nonempty sets $V_{1}$ and $V_{2}$ such that two vertices $x$ and $y$ are adjacent if and only if $x \in V_{1}$ and $y \in V_{2}$. If $\left|V_{1}\right|=m$ and $\left|V_{2}\right|=n$, such a graph is denoted $K_{m, n}$. For example, see $K_{3,4}$ in Figure 3.

Theorem 3. The betweenness centrality of a vertex in a complete bipartite graph $K_{m, n}$ is given by

$$
C_{B}(v)= \begin{cases}\frac{1}{m}\left(\begin{array}{l}
n \\
2
\end{array}\right), & \text { if } \operatorname{deg}(v)=n, \\
\frac{1}{n}\left(\begin{array}{c}
m \\
2
\end{array}\right), & \text { if } \operatorname{deg}(v)=m .\end{cases}
$$




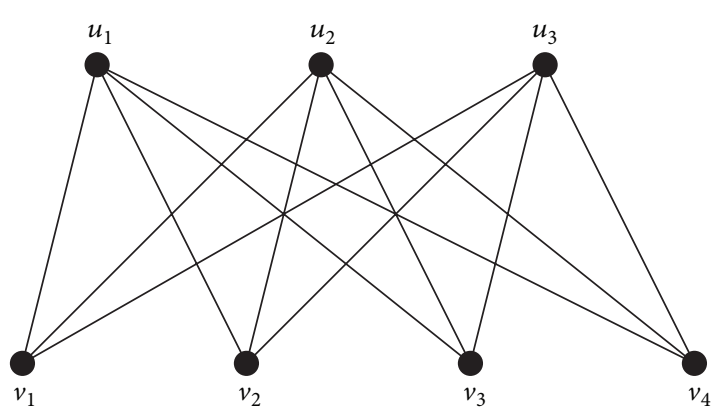

Figure 3: Complete bipartite graph $K_{3,4}$.

Proof. Consider a complete bipartite graph $K_{m, n}$ with a bipartition $\{U, W\}$ where $U=\left\{u_{1}, u_{2}, \ldots, u_{m}\right\}$ and $W=$ $\left\{w_{1}, w_{2}, \ldots, w_{n}\right\}$. The distance between any two vertices in $U$ (or in $W$ ) is 2 . Consider a vertex $u \in U$. Now any pair of vertices in $W$ contributes a betweenness centrality $1 / m$ to $u$. Since there are $\left(\begin{array}{l}n \\ 2\end{array}\right)$ pairs of vertices in $W, C_{B}(u)=(1 / m)\left(\begin{array}{l}n \\ 2\end{array}\right)$. In a similar way it can be shown that, for any vertex $w$ in $W$, $C_{B}(w)=(1 / n)\left(\begin{array}{c}m \\ 2\end{array}\right)$.

The relative centrality and graph centrality are as follows:

$$
\begin{aligned}
C_{B}^{\prime}(v) & =\frac{2 C_{B}(v)}{(m+n-1)(m+n-2)} \\
& = \begin{cases}\frac{2}{(m+n-1)(m+n-2)} & \begin{array}{l}
n \frac{1}{m}\left(\begin{array}{l}
n \\
2
\end{array}\right), \\
\frac{2}{(m+n-1)(m+n-2)}
\end{array} \\
C_{B}\left(K_{m, n}\right) & =\frac{\sum_{i=1}^{n}\left(\begin{array}{l}
\left.C_{B}^{\prime}\left(v^{*}\right)-C_{B}^{\prime}\left(v_{i}\right)\right] \\
2
\end{array}\right),}{(m+n-1)}, \operatorname{deg}(v)=n,\end{cases} \\
& = \begin{cases}\frac{m^{3}-n^{3}-\left(m^{2}-n^{2}\right)}{n(m+n-1)^{2}(m+n-2)}, & \text { if } m>n, \\
\frac{n^{2}(n-1)-m^{2}(m-1)}{m(m+n-1)^{2}(m+n-2)}, & \text { if } n>m .\end{cases}
\end{aligned}
$$

3.4. Betweenness Centrality of Vertices in Cocktail Party Graphs. The cocktail party graph $\mathrm{CP}(n)$ [17] is a unique regular graph of degree $2 n-2$ on $2 n$ vertices. It is obtained from $K_{2 n}$ by deleting a perfect matching (see Figure 4). The cocktail party graph of order $n$ is a complete $n$-partite graph with 2 vertices in each partition set. It is the graph complement of the ladder rung graph $L_{n}^{\prime}$ which is the graph union of $n$ copies of the path graph $P_{2}$ and the dual graph of the hypercube $Q_{n}[18]$.

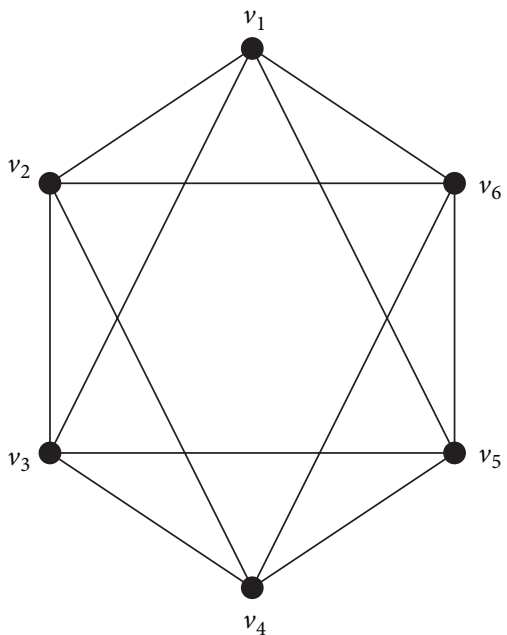

FIGURE 4: Cocktail party graph CP(3).

Theorem 4. The betweenness centrality of each vertex of a cocktail party graph of order $2 n$ is $1 / 2$.

Proof. Let the cocktail party graph $\mathrm{CP}(n)$ be obtained from the complete graph $K_{2 n}$ with vertices $\left\{v_{1}, \ldots\right.$, $\left.v_{n}, v_{n+1}, \ldots, v_{2 n}\right\}$ by deleting a perfect matching $\left\{\left(v_{1}, v_{n+1}\right)\right.$, $\left.\left(v_{2}, v_{n+2}\right), \ldots,\left(v_{n}, v_{2 n}\right)\right\}$. Now for each pair $\left(v_{i}, v_{n+i}\right)$ there is a geodesic of length 2 passing through each of the other $2 n-2$ vertices. Thus for any particular vertex, there are $n-1$ pairs of vertices of the above matching not containing that vertex giving a betweenness centrality $1 /(2 n-2)$ to that vertex. Therefore the betweenness centrality of any vertex is given by $(n-1) /(2 n-2)=1 / 2$.

The relative centrality and graph centrality are as follows:

$$
\begin{gathered}
C_{B}^{\prime}(v)=\frac{2 C_{B}(v)}{(2 n-1)(2 n-2)}=\frac{1}{(2 n-1)(2 n-2)}, \\
C_{B}(G)=0 .
\end{gathered}
$$

3.5. Betweenness Centrality of Vertices in Crown Graphs. The crown graph [18] is the unique $n-1$ regular graph with $2 n$ vertices obtained from a complete bipartite graph $K_{n, n}$ by deleting a perfect matching (see Figure 5). A crown graph on $2 n$ vertices can be viewed as an undirected graph with two sets of vertices $u_{i}$ and $v_{i}$ and with an edge from $u_{i}$ to $v_{j}$ whenever $i \neq j$. It is the graph complement of the ladder graph $L_{2 n}$. The crown graph is a distance-transitive graph.

Theorem 5. The betweenness centrality of each vertex of a crown graph of order $2 n$ is $(n+1) / 2$.

Proof. Let the crown graph be the complete bipartite graph $K_{n, n}$ with vertices $\left\{u_{1}, \ldots, u_{n}, v_{1}, \ldots, v_{n}\right\}$ minus a perfect matching $\left\{\left(u_{1}, v_{1}\right),\left(u_{2}, v_{2}\right), \ldots,\left(u_{n}, v_{n}\right)\right\}$. Consider any vertex; say $u_{1}$. Now for each pair $\left(u_{i}, v_{i}\right)$ other than $\left(u_{1}, v_{1}\right)$ there are $n-2$ paths of length 3 passing through $u_{1}$ out of $(n-1)(n-2)$ paths joining $u_{i}$ and $v_{i}$. Since there are $n-1$ such pairs, they 


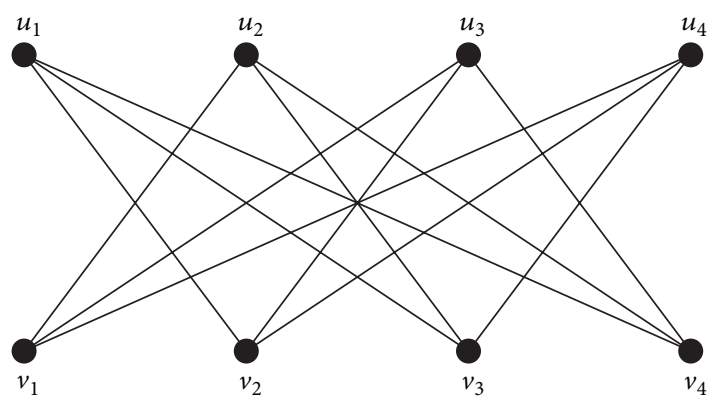

Figure 5: Crown graph with 8 vertices.

give $v_{1}$ the betweenness centrality $(n-1) \times((n-2) /(n-$ $1)(n-2))=1$. Again for each pair from $\left\{v_{2}, v_{3}, v_{4}, \ldots, v_{n}\right\}$ there exists exactly one path passing through $v_{1}$ out of $n-$ 2 paths. Since there are $\left(\begin{array}{c}n-1 \\ 2\end{array}\right)$ such pairs, they give $v_{1}$ the betweenness centrality $\left(\begin{array}{c}n-1 \\ 2\end{array}\right)(1 /(n-2))=(n-1) / 2$. Therefore the betweenness centrality of $v_{1}$ is given by $1+(n-1) / 2=$ $(n+1) / 2$. Since the graph is vertex transitive, the betweenness centrality of any vertex is given by $(n+1) / 2$.

The relative centrality and graph centrality are as follows:

$$
\begin{gathered}
C_{B}^{\prime}(v)=\frac{2 C_{B}(v)}{(2 n-1)(2 n-2)}=\frac{n+1}{(2 n-1)(2 n-2)}, \\
C_{B}(G)=0 .
\end{gathered}
$$

\subsection{Betweenness Centrality of Vertices in Paths}

Theorem 6. The betweenness centrality of any vertex in a path graph is the product of the number of vertices on either side of that vertex in the path.

Proof. Consider a path graph $P_{n}$ of $n$ vertices $\left\{v_{1}, v_{2}, \ldots, v_{n}\right\}$ (see Figure 6). Take a vertex $v_{k}$ in $P_{n}$. Then there are $k-1$ vertices on one side and $n-k$ vertices on the other side of $v_{k}$. Consequently there are $(k-1) \times(n-k)$ number of geodesics containing $v_{k}$. Hence $C_{B}\left(v_{k}\right)=(k-1)(n-k)$.

Note that, by symmetry, vertices at equal distance away from both ends of $P_{n}$ have the same centrality and it is maximum at the central vertex (vertices) and minimum at the end vertices. Consider

$$
\operatorname{Max} C_{B}\left(v_{k}\right)= \begin{cases}\frac{n(n-2)}{4}, & \text { when } n \text { is even, } \\ \frac{(n-1)^{2}}{4}, & \text { when } n \text { is odd. }\end{cases}
$$

Relative centrality of any vertex $v_{k}$ is given by

$$
C_{B}^{\prime}\left(v_{k}\right)=\frac{2 C_{B}\left(v_{k}\right)}{(n-1)(n-2)}=\frac{2(k-1)(n-k)}{(n-1)(n-2)} .
$$

Corollary 7. Graph centrality of $P_{n}$ is given by

$$
C_{B}\left(P_{n}\right)= \begin{cases}\frac{n(n+1)}{6(n-1)(n-2)}, & \text { if } n \text { is odd } \\ \frac{n(n+2)}{6(n-1)^{2}}, & \text { if } n \text { is even. }\end{cases}
$$

Proof. When $n$ is even, by definition

$$
\begin{aligned}
C_{B}\left(P_{n}\right)= & \frac{2}{(n-1)^{2}(n-2)} \sum_{i=1}^{n}\left[C_{B}\left(v^{*}\right)-C_{B}\left(v_{i}\right)\right] \\
= & \frac{4}{(n-1)^{2}(n-2)} \\
& \times\left\{\left[\frac{n(n-2)}{4}-0\right]+\left[\frac{n(n-2)}{4}-1 \cdot(n-2)\right]\right. \\
& \left.+\cdots+\left[\frac{n(n-2)}{4}-\left(\frac{n-4}{2}\right)\left(n-\frac{n-2}{2}\right)\right]\right\} \\
= & \frac{4}{(n-1)^{2}(n-2)} \\
& \times\left\{\left[\frac{n(n-2)}{4} \times \frac{n-2}{2}\right]\right. \\
= & \frac{-[1(n-2)+2(n-3)}{(n-1)^{2}(n-2)} \\
& \times\left\{\frac{n(n-2)^{2}}{8}-n \sum_{k=1}^{(n-4) / 2} k+\sum_{k=1}^{(n-4) / 2} k(k+1)\right\} \\
= & \left.\left.\frac{n(n+2)}{(n-1)^{2}(n-2)}\left(\frac{1}{4-1}\right)\left(n-\frac{n-2}{2}\right)\right]\right\} \\
& \times\left\{\frac{n(n-2)}{2}-\frac{n(n-4)}{2}\right. \\
& \times\left\{\frac{n(n-2)^{2}}{8}-\frac{n(n-2)(n-4)}{8}\right. \\
& \left.+\frac{(n-2)(n-4)}{8}+\frac{(n-2)(n-3)(n-4)}{24}\right\} \\
& \\
& \\
& \\
&
\end{aligned}
$$




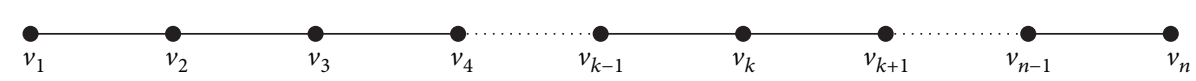

Figure 6: Path graph $P_{n}$.

When $n$ is odd, by definition

$$
\begin{aligned}
& C_{B}\left(P_{n}\right)=\frac{2}{(n-1)^{2}(n-2)} \sum_{i=1}^{n}\left[C_{B}\left(v^{*}\right)-C_{B}\left(v_{i}\right)\right] \\
& =\frac{4}{(n-1)^{2}(n-2)} \\
& \times\left\{\left[\frac{(n-1)^{2}}{4}-0\right]+\left[\frac{(n-1)^{2}}{4}-1 \cdot(n-2)\right]\right. \\
& \left.+\cdots+\left[\frac{(n-1)^{2}}{4}-\left(\frac{n-3}{2}\right)\left(n-\frac{n-1}{2}\right)\right]\right\} \\
& =\frac{4}{(n-1)^{2}(n-2)} \\
& \times\left\{\left[\frac{(n-1)^{2}}{4} \times \frac{n-1}{2}\right]\right. \\
& -[1(n-2)+2(n-3) \\
& \left.\left.+\cdots+\left(\frac{n-3}{2}\right)\left(n-\frac{n-1}{2}\right)\right]\right\} \\
& =\frac{4}{(n-1)^{2}(n-2)} \\
& \times\left\{\frac{(n-1)^{3}}{8}-n \sum_{k=1}^{(n-3) / 2} k+\sum_{k=1}^{(n-3) / 2} k(k+1)\right\} \\
& =\frac{4}{(n-1)^{2}(n-2)} \\
& \times\left\{\frac{(n-1)^{3}}{8}-\frac{n(n-1)(n-3)}{8}\right. \\
& \left.+\frac{(n-1)(n+1)(n-3)}{24}\right\} \\
& =\frac{1}{(n-1)(n-2)} \\
& \times\left\{\frac{(n-1)^{2}}{2}-\frac{n(n-3)}{2}+\frac{(n+1)(n-3)}{6}\right\} \\
& =\frac{n(n+1)}{6(n-1)(n-2)} \text {. }
\end{aligned}
$$

3.7. Betweenness Centrality of Vertices in Ladder Graphs. The ladder graph $L_{n}[19,20]$ is defined as the Cartesian product

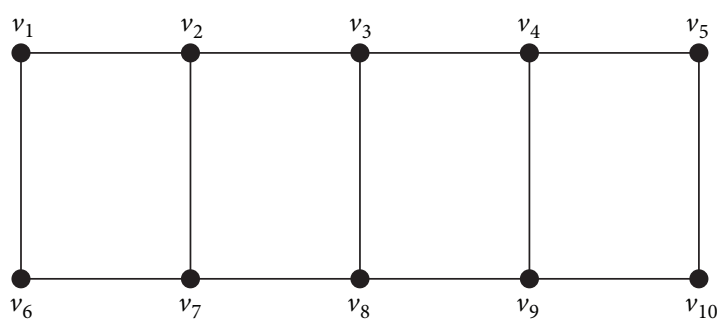

FIGURE 7: Ladder graph $L_{5}$.

$P_{2} \times P_{n}$ (see Figure 7). It is a planar undirected graph with $2 n$ vertices and $n+2(n-1)$ edges.

Theorem 8. The betweenness centrality of a vertex in a ladder graph $L_{n}$ is given by

$$
\begin{aligned}
C_{B}\left(v_{k}\right)= & (k-1)(n-k)+\sum_{j=0}^{k-1} \sum_{i=1}^{n-k} \frac{k-j}{k-j+i} \\
& +\sum_{j=0}^{k-2} \sum_{i=0}^{n-k} \frac{i+1}{k-j+i}, \quad 1 \leq k \leq n .
\end{aligned}
$$

Proof. By symmetry, let $v_{k}$ be any vertex such that $1 \leq k \leq$ $(n+1) / 2$. Consider the paths (in Figure 8) from upper left vertices $\left\{v_{1}, \ldots, v_{k-1}\right\}$ to upper right vertices $\left\{v_{k+1}, \ldots, v_{n}\right\}$ which give the betweenness centrality

$$
(k-1)(n-k)
$$

Now consider the paths from lower left vertices $\left\{v_{n+1}\right.$, $\left.\ldots, v_{n+k}\right\}$ to the upper right vertices $\left\{v_{k+1}, \ldots, v_{n}\right\}$ of $v_{k}$ which give the betweenness centrality

$$
\left.\begin{array}{c}
k\left\{\frac{1}{k+1}+\frac{1}{k+2}+\cdots+\frac{1}{n}\right\} \\
(k-1)\left\{\frac{1}{k}+\frac{1}{k+1}+\cdots+\frac{1}{n-1}\right\} \\
\left\{\frac{1}{2}+\frac{1}{3}+\cdots+\frac{1}{n-(k-1)}\right\}
\end{array}\right\}=\sum_{j=0}^{k-1} \sum_{i=1}^{n-k} \frac{k-j}{k-j+i} .
$$




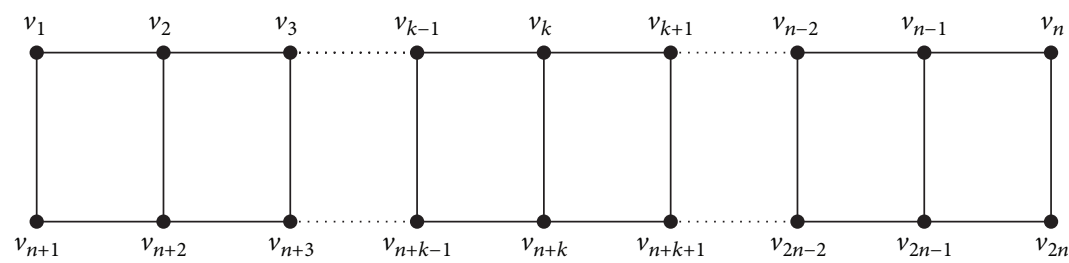

FIgURE 8: Ladder graph $L_{n}$.

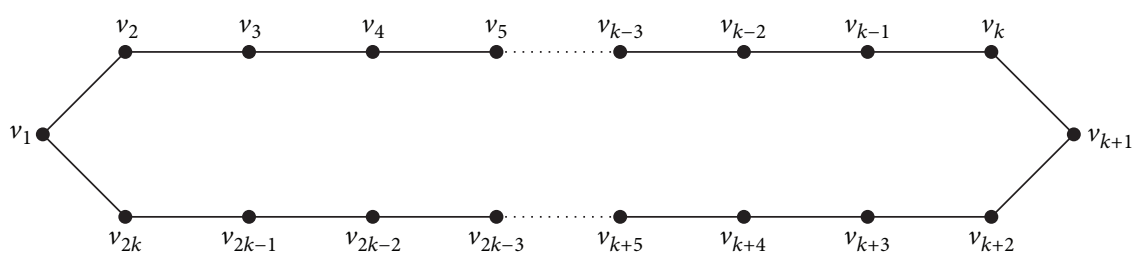

FIgURE 9: Even cycle with $2 k$ vertices.

Again consider the paths from upper left vertices $\left\{v_{1}, \ldots, v_{k-1}\right\}$ to the lower right vertices $\left\{v_{n+k}, \ldots, v_{2 n}\right\}$ of $v_{k}$ which give the betweenness centrality

$$
\begin{aligned}
& \left\{\frac{1}{k}+\frac{2}{k+1}+\cdots+\frac{n-(k-1)}{n}\right\}
\end{aligned}
$$

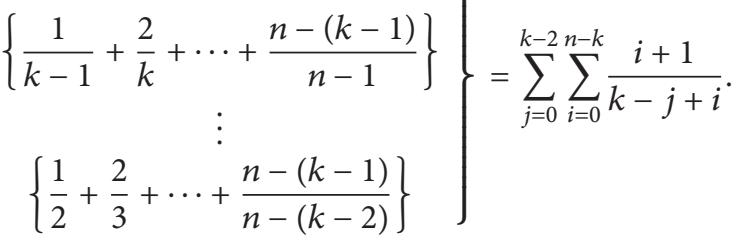

The above three equations when combined get the result.

\subsection{Betweenness Centrality of Vertices in Cycles}

Theorem 9. The betweenness centrality of a vertex in a cycle $C_{n}$ is given by

$$
C_{B}(v)= \begin{cases}\frac{(n-2)^{2}}{8}, & \text { if } n \text { is even, } \\ \frac{(n-1)(n-3)}{8}, & \text { if } n \text { is odd. }\end{cases}
$$

Proof.

Case 1 (when $n$ is even). Let $n=2 k, k \in \mathbb{Z}^{+}$, and let $C_{n}=\left(v_{1}, v_{2}, \ldots, v_{2 k}\right)$ be the given cycle. Consider a vertex $v_{1}$ (see Figure 9). Then $v_{k+1}$ is its antipodal vertex and there is no geodesic from $v_{k+1}$ to any other vertex passing through $v_{1}$. Hence we omit the pair $\left(v_{1}, v_{k+1}\right)$. Consider other pairs of antipodal vertices $\left(v_{i}, v_{k+i}\right)$ for $i=2,3, \ldots, k$. For each pair of these antipodal vertices there exist two paths of the same length $k$ and one of them contains $v_{1}$. Thus each pair contributes $1 / 2$ to the centrality of $v_{1}$ which gives a total of $(1 / 2)(k-1)$. Now consider all paths of length less than $k$ containing $v_{1}$. There are $k-i$ paths joining $v_{i}$ to vertices from $v_{2 k}$ to $v_{k+1+i}$ passing through $v_{1}$ for $i=2,3, \ldots, k-1$ and each contributes centrality 1 to $v_{1}$ giving a total $\sum_{i=2}^{k-1}(k-i)=$ $(k-1)(k-2) / 2$. Therefore the betweenness centrality of $v_{1}$ is $(1 / 2)(k-1)+(k-1)(k-2) / 2=(1 / 2)(k-1)^{2}=(1 / 8)(n-2)^{2}$. Since $C_{n}$ is vertex transitive, the betweenness centrality of any vertex is given by $(1 / 8)(n-2)^{2}$.

Case 2 (when $n$ is odd). Let $n=2 k+1, k \in \mathbb{Z}^{+}$, and let $C_{n}=\left(v_{1}, v_{2}, \ldots, v_{2 k+1}\right)$ be the given cycle. Consider a vertex $v_{1}$ (see Figure 10). Then $v_{k+1}$ and $v_{k+2}$ are its antipodal vertices at a distance $k$ from $v_{1}$ and there is no geodesic path from the vertexes $v_{k+1}$ and $v_{k+2}$ to any other vertex passing through $v_{1}$. Hence we omit $v_{1}$ and the pair $\left(v_{k+1}, v_{k+2}\right)$. Now consider paths of length $\leq k$ passing through $v_{1}$. There are $k+1-i$ paths joining $v_{i}$ to vertices from $v_{2 k+1}$ to $v_{k+1+i}$ passing through $v_{1}$ for $i=2,3, \ldots, k$ and each contributes a betweenness centrality 1 to $v_{1}$ giving a total of $\sum_{i=2}^{k}(k+1-i)=k(k-1) / 2=$ $(n-1)(n-3) / 8$. Since $C_{n}$ is vertex transitive, the betweenness centrality of any vertex is given by $(n-1)(n-3) / 8$.

The relative centrality and graph centrality are as follows:

$$
\begin{aligned}
C_{B}^{\prime}(v)= & \frac{C_{B}(v)}{\operatorname{Max} C_{B}(v)}=\frac{2 C_{B}(v)}{(n-1)(n-2)} \\
= & \begin{cases}\frac{n-2}{4(n-1)}, & \text { if } n \text { is even, } \\
\frac{n-3}{4(n-2)}, & \text { if } n \text { is odd, } \\
C_{B}\left(C_{n}\right)=0 .\end{cases}
\end{aligned}
$$

3.9. Betweenness Centrality of Vertices in Circular Ladder Graphs $\mathrm{CL}_{n}$. The circular ladder graph $\mathrm{CL}_{n}$ consists of two concentric $n$-cycles in which each pair of the $n$ corresponding vertices is joined by an edge (see Figure 11). It is a 3-regular simple graph isomorphic to the Cartesian product $K_{2} \times C_{n}$. 


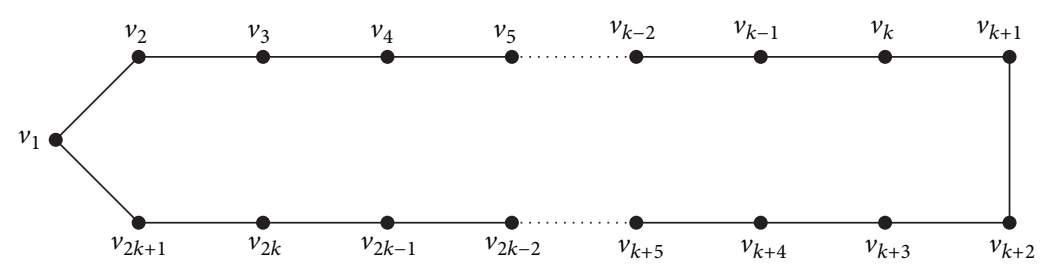

FIGURE 10: Odd cycle with $2 k+1$ vertices.

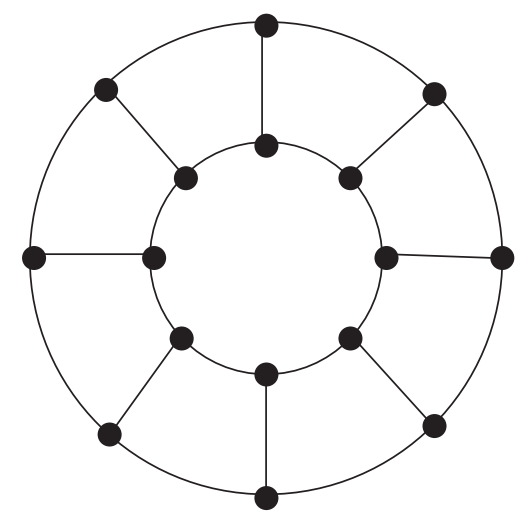

Figure 11: Circular ladder.

Theorem 10. The betweenness centrality of a vertex in a circular ladder $C L_{n}$ is given by

$$
C_{B}(v)= \begin{cases}\frac{(n-1)^{2}+1}{4}, & \text { when } n \text { is even, } \\ \frac{(n-1)^{2}}{4}, & \text { when } n \text { is odd } .\end{cases}
$$

Proof.

Case 1 (when $n$ is even). Let $n=2 k, k \in \mathbb{Z}^{+}$. Let $C_{2 k}=$ $\left(u_{1}, u_{2}, \ldots, u_{2 k}\right)$ be the outer cycle and $C_{2 k}^{\prime}=\left(v_{1}, v_{2}, \ldots, v_{2 k}\right)$ the inner cycle. Consider any vertex; say $u_{1}$ in $C_{2 k}$. Then its betweenness centrality as a vertex in $C_{2 k}$ is $(k-1)^{2} / 2$. Now the geodesics from outer vertices $u_{i}$ to the inner vertices $v_{1}, v_{2 k}, \ldots, v_{k+i}$ for $i=2, \ldots, k$ (see Figure 12) and from $u_{2 k+2-i}$ to $v_{1}, v_{2}, \ldots, v_{k+2-i}$ for $i=2, \ldots, k$ by symmetry contribute to $u_{1}$ the betweenness centrality

$$
\begin{array}{r}
2 \sum_{i=2}^{k}\left(\frac{1}{i}+\frac{2}{i+1}+\cdots+\frac{k+1-i}{k}+\frac{k+2-i}{2 k+2}\right) \\
=2\left(\frac{1}{2}+\frac{1+2}{3}+\cdots+\frac{1+2+\cdots+k-1}{k}\right. \\
\left.\quad+\frac{2+3+\cdots+k}{2 k+2}\right) \\
=2 \sum_{p=2}^{k} \frac{(1+2+\cdots+p-1)}{p}+\frac{k(k+1)-2}{2(k+1)} \\
=2 \sum_{p=2}^{k} \frac{p-1}{2}+\frac{k(k+1)-2}{2(k+1)}=\frac{k^{2}}{2}-\frac{1}{k+1} .
\end{array}
$$

Again the pair $\left(u_{k+1}, v_{1}\right)$ contributes to $u_{1}$ the betweenness centrality $2 /(2 k+2)$. Therefore the betweenness centrality of $u_{1}$ is given as

$$
\begin{aligned}
C_{B}\left(u_{1}\right) & =\frac{(k-1)^{2}}{2}+\frac{k^{2}}{2}-\frac{1}{k+1}+\frac{1}{k+1} \\
& =\frac{(k-1)^{2}}{2}+\frac{k^{2}}{2}=\frac{(2 k-1)^{2}+1}{4} \\
& =\frac{(n-1)^{2}+1}{4}
\end{aligned}
$$

Case 2 (when $n$ is odd). Let $n=2 k+1, k \in \mathbb{Z}^{+}$. Let $C_{2 k+1}=\left(u_{1}, u_{2}, \ldots, u_{2 k+1}\right)$ be the outer cycle and $C_{2 k+1}^{\prime}=$ $\left(v_{1}, v_{2}, \ldots, v_{2 k+1}\right)$ the inner cycle. Consider any vertex; say $u_{1}$ in $C_{2 k+1}$. Then its betweenness centrality as a vertex in $C_{2 k+1}$ is $k(k-1) / 2$. Now consider the geodesics from outer vertices $u_{i}$ to the inner vertices $v_{1}, v_{2 k+1}, \ldots, v_{k+i+1}$ for $i=2, \ldots, k+1$ (see Figure 13) and from $u_{2 k+3-i}$ to $v_{1}, v_{2}, \ldots, v_{k+2-i}$ for $i=$ $2,3, \ldots, k+1$ which give a betweenness centrality

$$
\begin{aligned}
& 2 \sum_{i=2}^{k}\left(\frac{1}{i}+\frac{2}{i+1}+\cdots+\frac{k+2-i}{k+1}\right) \\
& \quad=2\left(\frac{1}{2}+\frac{1+2}{3}+\cdots+\frac{1+2+\cdots+k}{k+1}\right) \\
& \quad=2 \sum_{p=2}^{k+1} \frac{(1+2+\cdots+p-1)}{p} \\
& \quad=2 \sum_{p=2}^{k+1} \frac{p-1}{2}=\frac{k(k+1)}{2} .
\end{aligned}
$$

Therefore the betweenness centrality of $u_{1}$ is given as

$$
C_{B}\left(u_{1}\right)=\frac{k(k-1)}{2}+\frac{k(k+1)}{2}=k^{2}=\frac{(n-1)^{2}}{4} \text {. }
$$

The relative centrality and graph centrality are as follows:

$$
\begin{aligned}
C_{B}^{\prime}(v) & =\frac{2 C_{B}(v)}{(2 n-1)(2 n-2)} \\
& = \begin{cases}\frac{(n-1)^{2}+1}{2(2 n-1)(2 n-2)}, & \text { when } n \text { is even, } \\
\frac{(n-1)}{4(2 n-1)}, & \text { when } n \text { is odd, } \\
C_{B}(G)=0 .\end{cases}
\end{aligned}
$$



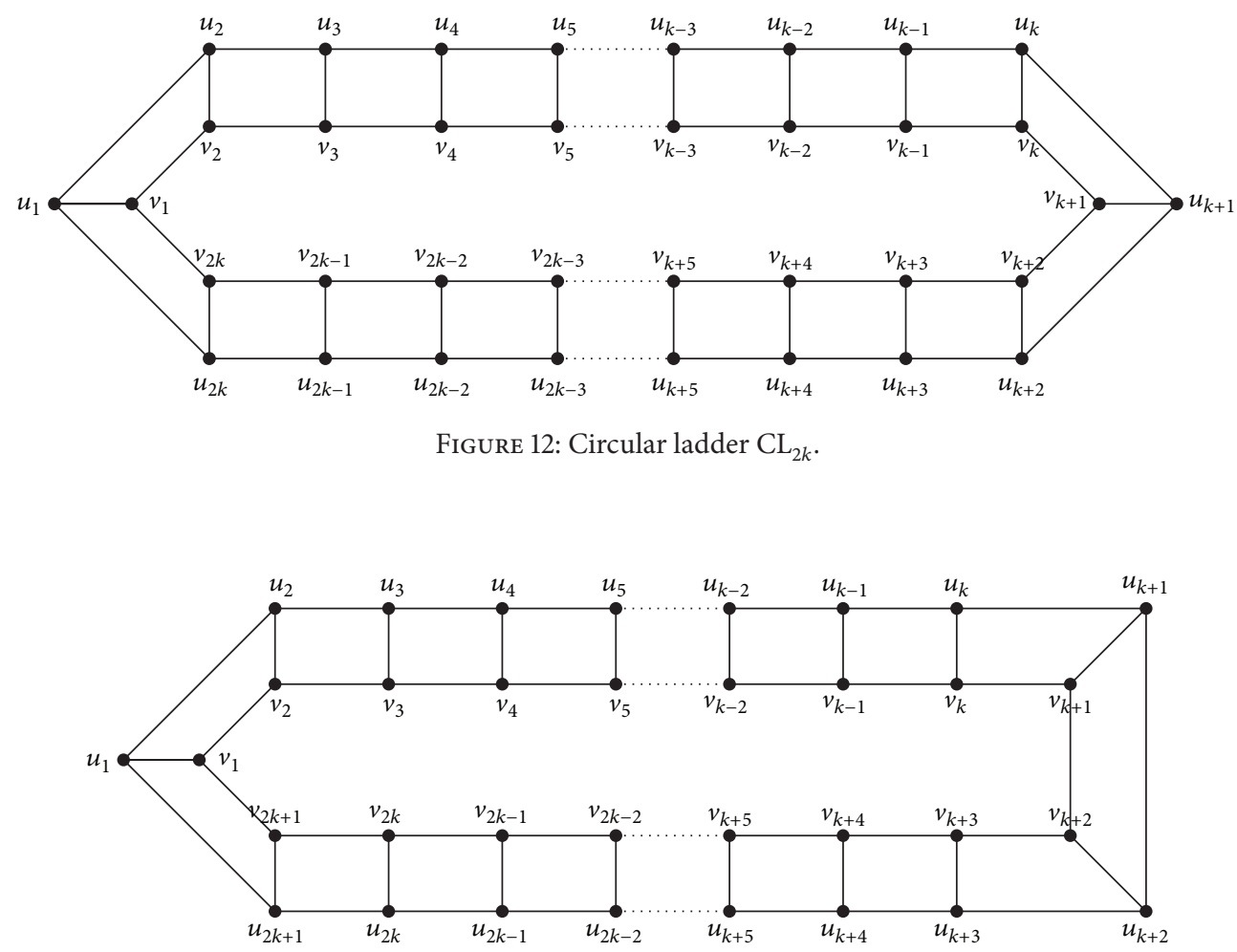

Figure 13: Circular ladder $\mathrm{CL}_{2 k+1}$.

3.10. Betweenness Centrality of Vertices in Trees. In a tree, there is exactly one path between any two vertices. Therefore the betweenness centrality of a vertex is the number of paths passing through that vertex. A branch at a vertex $v$ of a tree $T$ is a maximal subtree containing $v$ as an end vertex. The number of branches at $v$ is $\operatorname{deg}(v)$.

Theorem 11. The betweenness centrality $C_{B}(v)$ of a vertex $v$ in a tree $T$ is given by

$$
\mathscr{C}\left(n_{1}, n_{2}, \ldots, n_{k}\right)=\sum_{i<j} n_{i} n_{j}
$$

where the arguments $n_{i}$ denote the number of vertices of the branches at $v$ excluding $v$, taken in any order.

Proof. Consider a vertex $v$ in a tree $T$. Let there be $k$ branches with number of vertices $n_{1}, n_{2}, \ldots, n_{k}$ excluding $v$. The betweenness centrality of $v$ in $T$ is the total number of paths passing through $v$. Since all the branches have only one vertex $v$ in common, excluding $v$, every path joining a pair of vertices of different branches passes through $v$. Thus the total number of such pairs gives the betweenness centrality of $v$. Hence $\mathscr{C}=\sum_{i<j} n_{i} n_{j}$.

Example 12. Consider the tree given in Figure 14.

Here

$$
\begin{aligned}
& C_{B}\left(v_{1}\right)=C_{B}\left(v_{4}\right)=C_{B}\left(v_{6}\right)=C_{B}\left(v_{7}\right)=\mathscr{C}(6)=0, \\
& C_{B}\left(v_{2}\right)=\mathscr{C}(1,3,2)=11,
\end{aligned}
$$

$$
\begin{aligned}
& C_{B}\left(v_{3}\right)=\mathscr{C}(5,1)=5, \\
& C_{B}\left(v_{5}\right)=\mathscr{C}(1,1,4)=9 .
\end{aligned}
$$

Example 13. Table 2 gives the possible values for the betweenness centrality of a vertex in a tree of 9 vertices.

We consider the different possible combinations of the arguments in $\mathscr{C}$ so that the sum of arguments is 8 .

3.11. Betweenness Centrality of Vertices in Hypercubes. The $n$ cube or $n$-dimensional hypercube $Q_{n}$ is defined recursively by $Q_{1}=K_{2}$ and $Q_{n}=K_{2} \times Q_{n-1}$. That is, $Q_{n}=\left(K_{2}\right)^{n}$ Harary [21]. $Q_{n}$ is an $n$-regular graph containing $2^{n}$ vertices and $n 2^{n-1}$ edges. Each vertex can be labeled as a string of $n$ bits 0 and 1 . Two vertices of $Q_{n}$ are adjacent if their binary representations differ at exactly one place (see Figure 15). The $2^{n}$ vertices are labeled by the $2^{n}$ binary numbers from 0 to $2^{n}-1$. By definition, the length of a path between two vertices $u$ and $v$ is the number of edges of the path. To move from $u$ to $v$ it suffices to cross successively the vertices whose labels are those obtained by changing the bits of $u$ one by one in order to transform $u$ into $v$. If $u$ and $v$ differ only in $i$ bits, the distance (hamming distance) between $u$ and $v$ denoted by $d(u, v)$ is $i$ $[22,23]$. For example, if $u=(101010)$ and $v=(110011)$, then $d(u, v)=3$.

There exists a path of length at most $n$ between any two vertices of $Q_{n}$. In other words an $n$-cube is a connected graph of diameter $n$. The number of geodesics between $u$ and $v$ is 


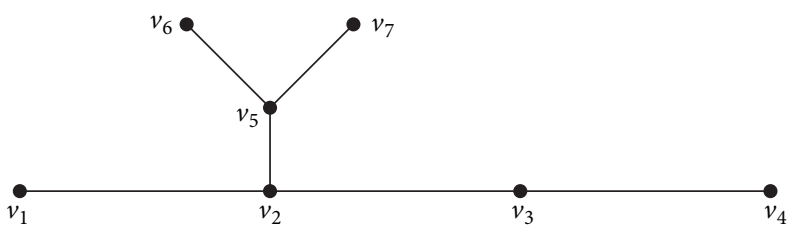

FIGURE 14: A tree with 7 vertices.
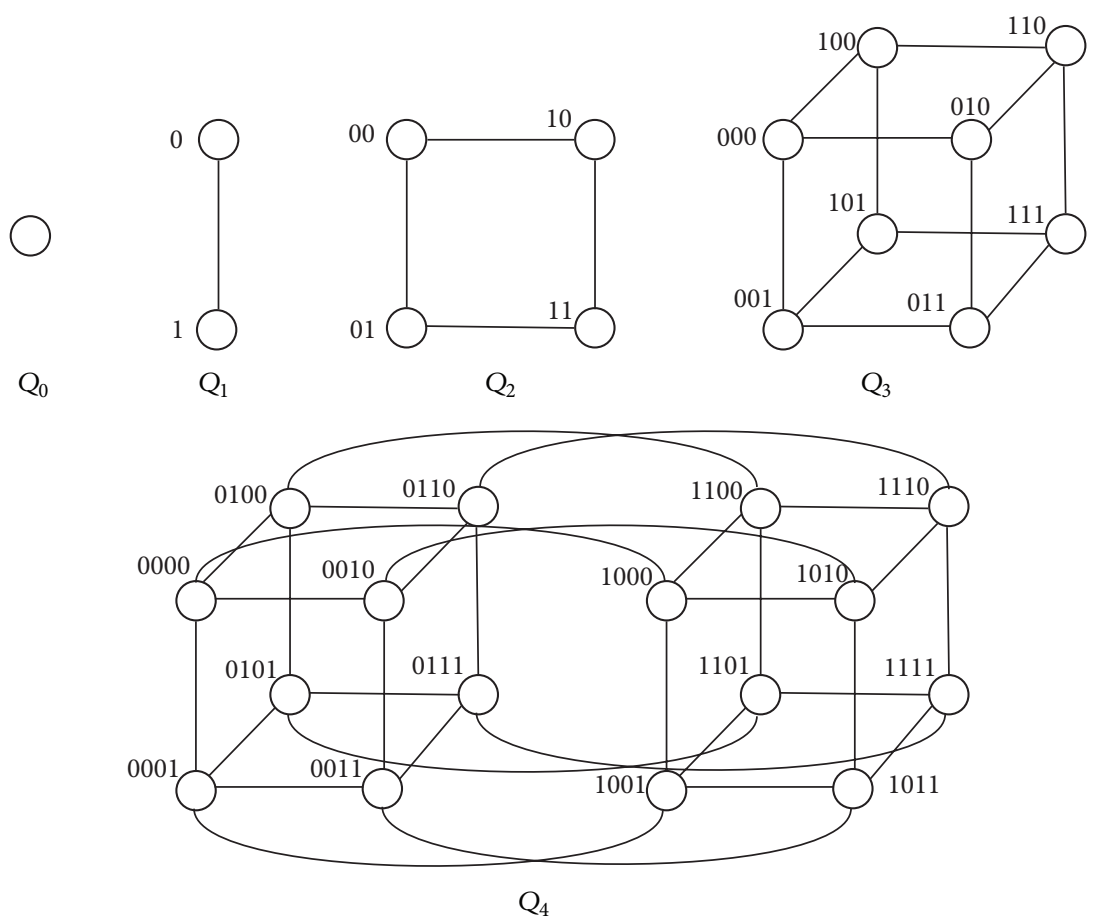

Figure 15: Hypercubes.

given by the number of permutations $d(u, v)$ !. A hypercube is bipartite and interval regular. For any two vertices $u$ and $v$, the interval $I(u, v)$ induces a hypercube of dimension $d(u, v)$ [24]. Another important property of $n$-cube is that it can be constructed recursively from lower dimensional cubes (see Figure 15). Consider two identical $(n-1)$-cubes. Each $(n-1)$ cube has $2^{n-1}$ vertices and each vertex has a labeling of $(n-1)$ bits. Join all identical pairs of vertices of the two cubes. Now increase the number of bits in the labels of all vertices by placing 0 in the $i$ th place of the first cube and 1 in the $i$ th place of second cube. Thus we get an $n$-cube with $2^{n}$ vertices, each vertex having a label of $n$-bits and the corresponding vertices of the two $(n-1)$-cubes differ only in the $i$ th bit. This $n$-cube so constructed can be seen as the union of $n$ pairs of $(n-1)$-cubes differing in exactly one position of bits. Thus the number of $(n-1)$-cubes in an $n$-cube is $2 n$. The operation of splitting an $n$-cube into two disjoint $(n-1)$-cubes so that the vertices of the two $(n-1)$-cubes are in a one-to-one correspondence will be referred to as tearing [23]. Since there are $n$ bits, there are $n$ directions for tearing. In general there are $\left(\begin{array}{l}n \\ k\end{array}\right) 2^{n-k}$ number of $k$-subcubes associated with an $n$-cube.
Theorem 14. The betweenness centrality of a vertex in a hypercube $Q_{n}$ is given by $2^{n-2}(n-2)+1 / 2$.

Proof. The hypercube $Q_{n}$ of dimension $n$ is a vertex transitive $n$-regular graph containing $2^{n}$ vertices. Each vertex can be written as an $n$ tuple of binary digits 0 and 1 with adjacent vertices differing in exactly one coordinate. The distance between two vertices $x$ and $y$ denoted by $(x, y)$ is the number of places in which the corresponding coordinates of $x$ and $y$ differ and the number of distinct geodesics between $x$ and $y$ is $d(x, y)$ ! [22]. Let $0=(0,0, \ldots, 0)$ be a vertex in $Q_{n}$ whose betweenness centrality has to be determined. Consider all $k$ subcubes containing the vertex 0 for $2 \leq k \leq n$. Each $k$ subcube has vertices with $n-k$ zeros in their labels. Since each $k$-subcube can be distinguished by $k$ coordinates, the number of $k$-subcubes containing the vertex 0 is $\left(\begin{array}{l}n \\ k\end{array}\right)$. The vertex 0 lies on a geodesic joining a pair of vertices if and only if the pair of vertices forms a pair of antipodal vertices of some subcube containing 0 [24]. So we consider all pairs of antipodal vertices excluding the vertex 0 and its antipodal vertex in each $k$-subcube containing 0 . If a vertex of a $k$ subcube has $r$ ones, then its antipodal vertex has $k-r$ ones. 
TABLE 2: Possible values for betweenness centrality in a tree of 9 vertices.

\begin{tabular}{|c|c|c|}
\hline Number of args. & Possible combinations & Values \\
\hline 8 & $\mathscr{C}(1,1,1,1,1,1,1,1)$ & 28 \\
\hline 7 & $\mathscr{C}(2,1,1,1,1,1,1)$ & 27 \\
\hline \multirow{2}{*}{6} & $\mathscr{C}(2,2,1,1,1,1)$ & 26 \\
\hline & $\mathscr{C}(3,1,1,1,1,1)$ & 25 \\
\hline \multirow{3}{*}{5} & $\mathscr{C}(2,2,2,1,1)$ & 25 \\
\hline & $\mathscr{C}(3,2,1,1,1)$ & 24 \\
\hline & $\mathscr{C}(4,1,1,1,1)$ & 22 \\
\hline \multirow{5}{*}{4} & $\mathscr{C}(2,2,2,2)$ & 24 \\
\hline & $\mathscr{C}(3,2,2,1)$ & 23 \\
\hline & $\mathscr{C}(3,3,1,1)$ & 22 \\
\hline & $\mathscr{C}(5,1,1,1)$ & 18 \\
\hline & $\mathscr{C}(4,2,1,1)$ & 21 \\
\hline \multirow{5}{*}{3} & $\mathscr{C}(3,3,2)$ & 21 \\
\hline & $\mathscr{C}(4,2,2)$ & 20 \\
\hline & $\mathscr{C}(4,3,1)$ & 19 \\
\hline & $\mathscr{C}(5,2,1)$ & 17 \\
\hline & $\mathscr{C}(6,1,1)$ & 13 \\
\hline \multirow{4}{*}{2} & $\mathscr{C}(4,4)$ & 16 \\
\hline & $\mathscr{C}(5,3)$ & 15 \\
\hline & $\mathscr{C}(6,2)$ & 12 \\
\hline & $\mathscr{C}(7,1)$ & 7 \\
\hline 1 & $\mathscr{C}(8)$ & 0 \\
\hline
\end{tabular}

For any pair of such antipodal vertices there are $k$ ! geodesics joining them and of that $r !(k-r)$ ! paths are passing through 0 . Thus each pair contributes $r !(k-r) ! / k !$, that is, $1 /\left(\begin{array}{l}k \\ r\end{array}\right)$, to the betweenness centrality of 0 .

By symmetry, when $k$ is even the number of distinct pairs of required antipodal vertices are given by $\left(\begin{array}{l}k \\ r\end{array}\right)$ for $1 \leq r<k / 2$ and $(1 / 2)\left(\begin{array}{l}k \\ r\end{array}\right)$ for $r=k / 2$. When $k$ is odd, the number of distinct pairs of required antipodal vertices is given by $\left(\begin{array}{l}k \\ r\end{array}\right)$ for $1 \leq r \leq(k-1) / 2$. Taking all such pairs of antipodal vertices in a $k$-subcube we get the contribution of betweenness centrality as $\sum_{r=1}^{k / 2-1}\left(\begin{array}{c}k \\ r\end{array}\right)\left(1 /\left(\begin{array}{c}k \\ r\end{array}\right)\right)+(1 / 2)\left(\begin{array}{c}k \\ k / 2\end{array}\right)\left(1 /\left(\begin{array}{c}k \\ k / 2\end{array}\right)\right)=(k-1) / 2$, when $k$ is even, and $\sum_{r=1}^{(k-1) / 2}\left(\begin{array}{c}k \\ r\end{array}\right)\left(1 /\left(\begin{array}{l}k \\ r\end{array}\right)\right)=(k-1) / 2$, when $k$ is odd. Therefore considering all $k$-subcubes for $2 \leq k \leq n$, we get the betweenness centrality of 0 as

$$
\begin{aligned}
C_{B}(0) & =\sum_{k=2}^{n}\left(\frac{k-1}{2}\right)\left(\begin{array}{l}
n \\
k
\end{array}\right) \\
& =\frac{1}{2}\left[\sum_{k=2}^{n} k\left(\begin{array}{l}
n \\
k
\end{array}\right)-\sum_{k=2}^{n}\left(\begin{array}{l}
n \\
k
\end{array}\right)\right] \\
& =2^{n-2}(n-2)+\frac{1}{2} .
\end{aligned}
$$

Therefore, for any vertex $v$,

$$
C_{B}(v)=2^{n-2}(n-2)+\frac{1}{2} .
$$

The relative centrality and graph centrality are as follows:

$$
\begin{aligned}
C_{B}^{\prime}(v) & =\frac{2 C_{B}(v)}{\left(2^{n}-1\right)\left(2^{n}-2\right)} \\
& =\frac{2^{n-1}(n-2)+1}{\left(2^{n}-1\right)\left(2^{n}-2\right)}, \quad C_{B}(G)=0 .
\end{aligned}
$$

\section{Conclusion}

Betweenness centrality is a useful metric for analyzing graph structures. When compared to other centrality measures, computation of betweenness centrality is rather difficult as it involves calculation of the shortest paths between all pairs of vertices in a graph. We have derived expressions for betweenness centrality of graphs which are the basic components of larger and complex networks. This study is therefore helpful for analysing larger classes of graphs.

\section{Conflict of Interests}

The authors declare that there is no conflict of interests regarding the publication of this paper.

\section{Acknowledgment}

This work was supported by the University Grants Commission (UGC), Government of India, under the scheme of Faculty Development Programme (FDP) for colleges.

\section{References}

[1] E. Otte and R. Rousseau, "Social network analysis: a powerful strategy, also for the information sciences," Journal of Information Science, vol. 28, no. 6, pp. 441-453, 2002.

[2] K. Park and A. Yilmaz, "A social network an analysis approach to analyze road networks," in Proceedings of the ASPRS Annual Conference, San Diego, Calif, USA, 2010.

[3] V. Latora and M. Marchiori, "A measure of centrality based on network efficiency," New Journal of Physics, vol. 9, article 188, 2007.

[4] E. Estrada, "Virtual identification of essential proteins within the protein interaction network of yeast," Proteomics, vol. 6, no. 1, pp. 35-40, 2006.

[5] L. C. Freeman, "Centrality in social networks conceptual clarification," Social Networks, vol. 1, no. 3, pp. 215-239, 1979.

[6] D. Koschützki and F. Schreiber, "Centrality analysis methods for biological networks and their application to gene regulatory networks," Gene Regulation and Systems Biology, vol. 2008, no. 2, pp. 193-201, 2008.

[7] A. M. M. González, B. Dalsgaard, and J. M. Olesen, "Centrality measures and the importance of generalist species in pollination networks," Ecological Complexity, vol. 7, no. 1, pp. 36-43, 2010.

[8] M. E. J. Newman, "Scientific collaboration networks. II. sho rtest paths, weighted networks, and centrality," Physical Review E, vol. 64, no. 1, pp. 1-7, 2001.

[9] M. Rubinov and O. Sporns, "Complex network measures of brain connectivity: uses and interpretations," NeuroImage, vol. 52, no. 3, pp. 1059-1069, 2010. 
[10] P. Bonacich, "Power and centrality: a family of measures," American Journal of Sociology, vol. 92, no. 5, pp. 1170-1182, 1987.

[11] S. P. Borgatti, "Centrality and network flow," Social Networks, vol. 27, no. 1, pp. 55-71, 2005.

[12] S. P. Borgatti and M. G. Everett, "A graph-theoretic perspective on centrality," Social Networks, vol. 28, no. 4, pp. 466-484, 2006.

[13] E. Estrada, D. J. Higham, and N. Hatano, "Communicability betweenness in complex networks," Physica A: Statistical Mechanics and its Applications, vol. 388, no. 5, pp. 764-774, 2009.

[14] S. Gago, J. Coroničová Hurajová, and T. Madaras, "On betweenness-uniform graphs," Czechoslovak Mathematical Journal, vol. 63, no. 3, pp. 629-642, 2013.

[15] A. Bavelas, "A mathematical model for group structure. Human organization," Applied Anthropology, vol. 7, no. 3, pp. 16-30, 1948.

[16] L. C. Freeman, "A set of measures of centrality based on betweenness," Sociometry, vol. 40, pp. 35-41, 1977.

[17] D. Cvetkovic, M. Doob, and S. Simic, "Generalized line graphs," Journal of Graph Theory, vol. 5, no. 4, pp. 385-399, 1981.

[18] N. Biggs, Algebraic Graph Theory, Cambridge University Press, Cambridge, UK, 1993.

[19] H. Hosoya and F. Harary, "On the matching properties of three fence graphs," Journal of Mathematical Chemistry, vol. 12, no. 1, pp. 211-218, 1993.

[20] M. Noy and A. Ribó, "Recursively constructible families of graphs," Advances in Applied Mathematics, vol. 32, no. 1-2, pp. 350-363, 2004.

[21] F. Harary, Graph Theory, Addison-Wesley, Reading, Mass, USA, 1969.

[22] S. Foldes, "A characterization of hypercubes," Discrete Mathematics, vol. 17, no. 2, pp. 155-159, 1977.

[23] Y. Saad and M. H. Schultz, "Topological properties of hypercubes," IEEE Transactions on Computers, vol. 37, no. 7, pp. 867$872,1988$.

[24] H. M. Mulder, "Interval-regular graphs," Discrete Mathematics, vol. 41, no. 3, pp. 253-269, 1982. 


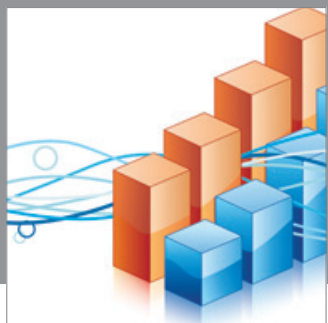

Advances in

Operations Research

mansans

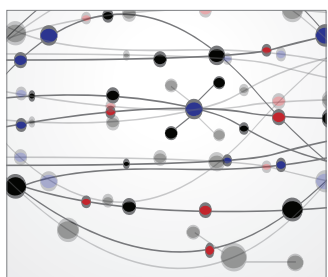

The Scientific World Journal
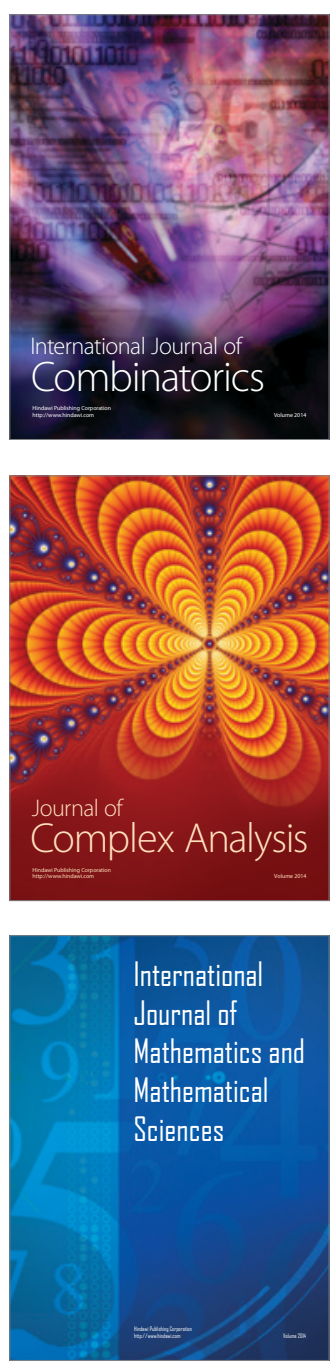
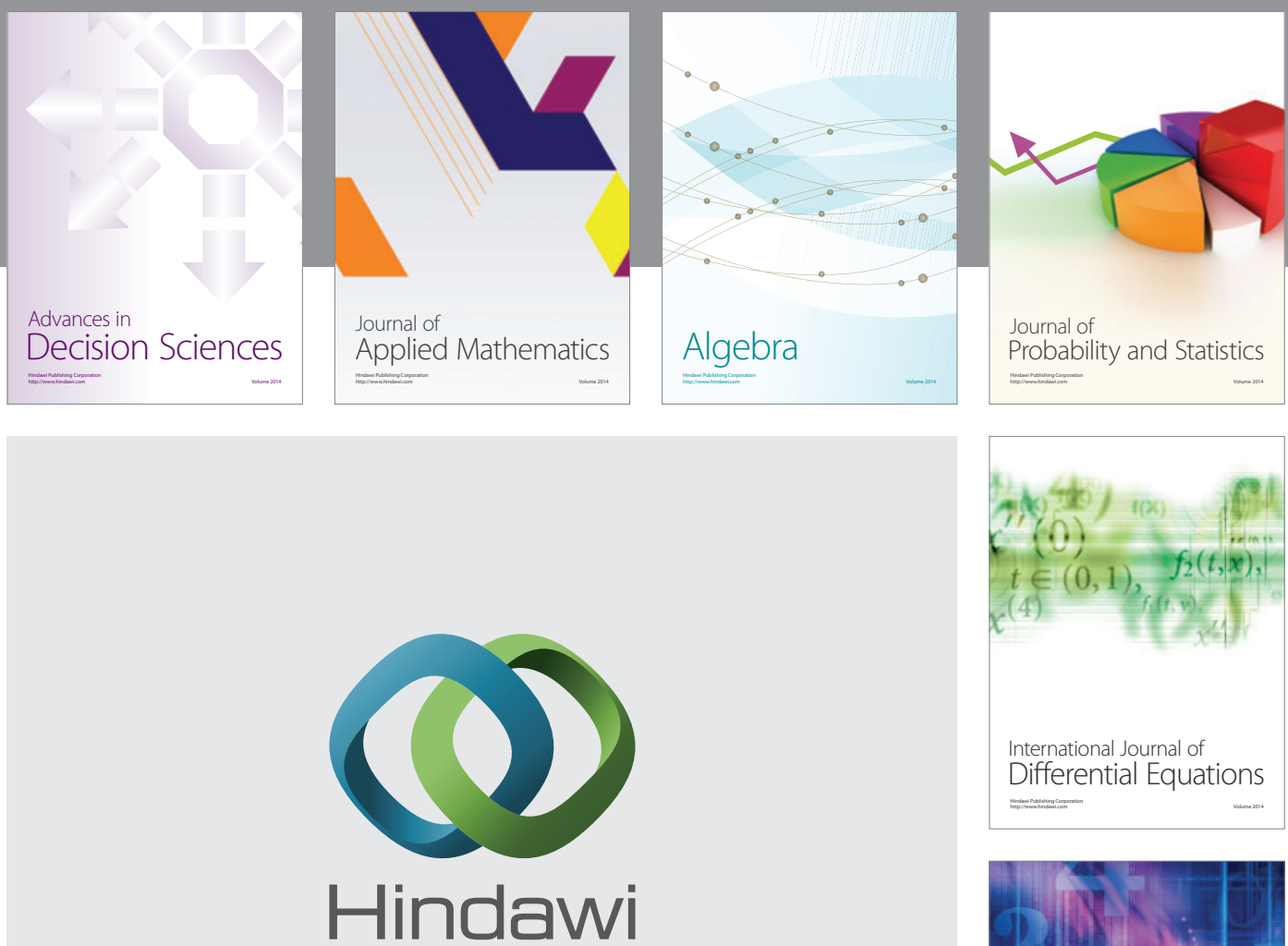

Submit your manuscripts at http://www.hindawi.com
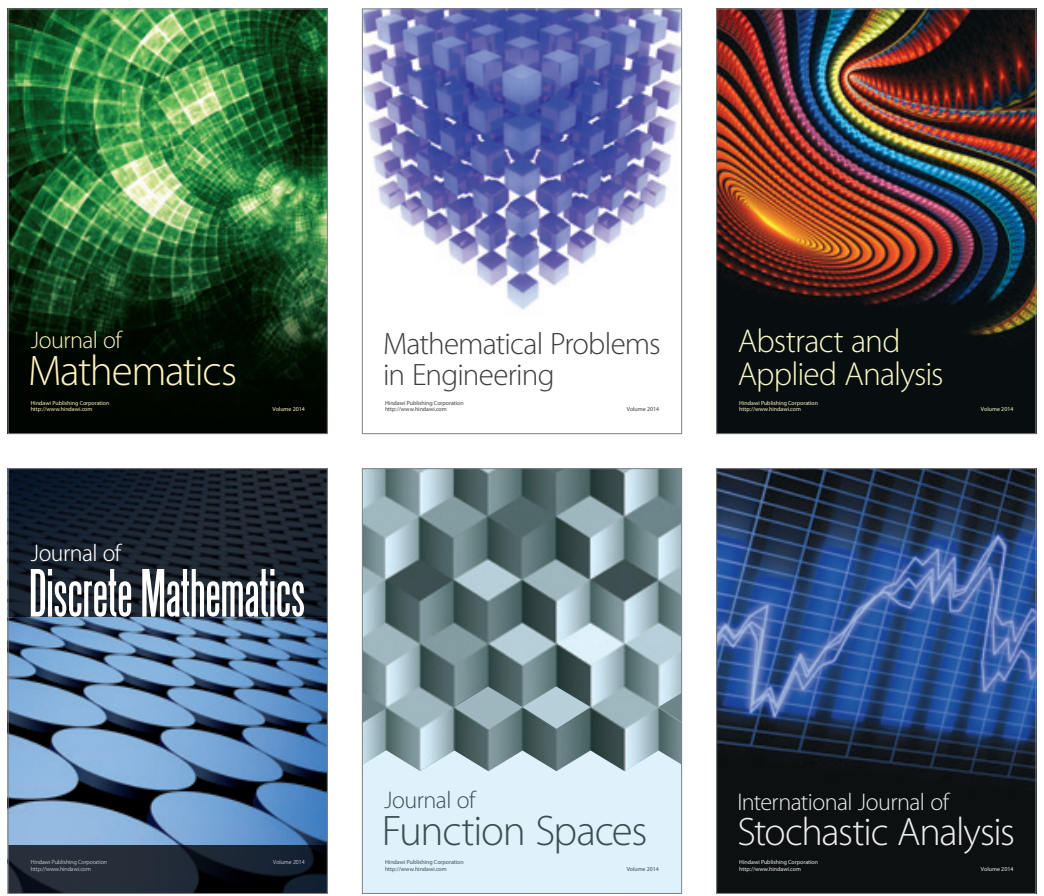

Journal of

Function Spaces

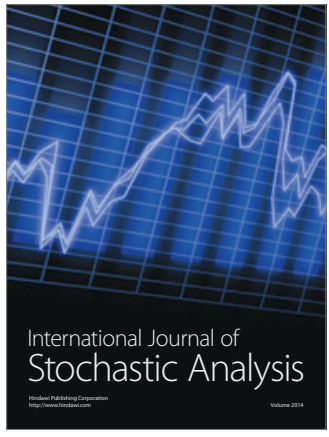

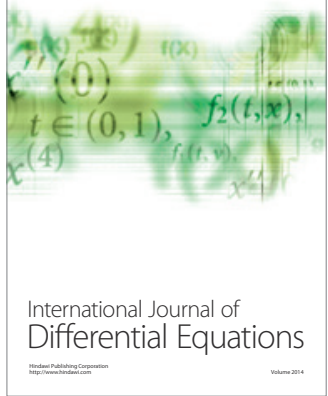
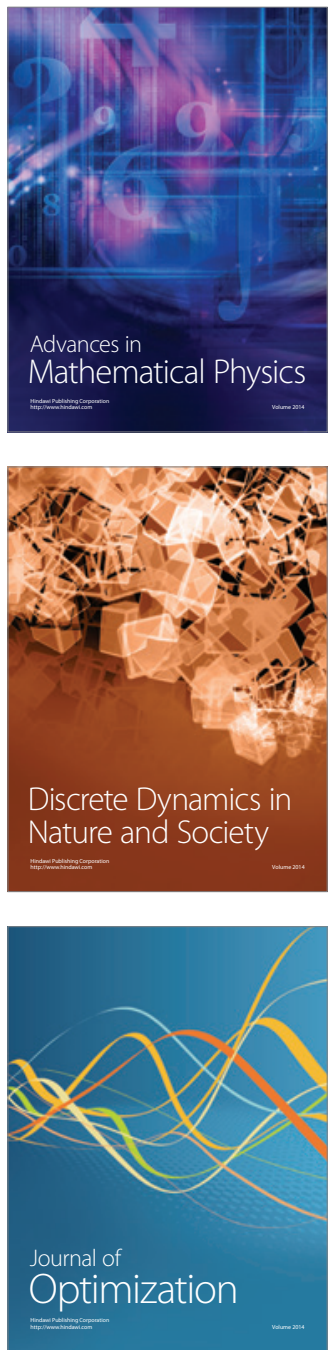